\title{
Adapting ECG Morphology Changes from Reduced-Lead Set by Specifically Trained Algorithms for Acute Ischemia Detection
}

\author{
JQ Xue \\ GE Healthcare, Wauwatosa, WI, USA
}

\begin{abstract}
The reduced lead set in this study consists of a subset of 12-lead set with all the same limb leads, plus $v 1$ and $v 5$ chest leads. They were then converted to 12-lead signals with a trained matrix. The aims of this study are to evaluate the changes of morphology due to the conversion and the possibility of adapting the changes with the new criteria trained with a large ischemia ECG database. For the evaluation, we first applied the standard 12 lead computerized algorithm to the converted 12-lead ECGs. We then applied 2 training algorithms to the converted 12-lead ECG to obtain the specific criteria. One algorithm is based on neural network models, and the other is based on the binary-tree classification model with automatically induced rules (C4.5). The study used the chest pain databases from Mayo Clinic and Medical College of Wisconsin, which include ECGs from 993 anterior MI patients, 1187 inferior MI patients and 1987 Nonischemic patients based on their final confirmation with Cathlab or Biomarker tests. The results show that, without retraining, the reduced-lead set achieved 53\% sensitivity for Inferior MI, compared with 54\% from standard 12 lead set, and with both specificity of $99 \%$. For anterior MI, the sensitivity of reduced lead set is $37 \%$, compared to the $46 \%$ for standard 12 lead. After retraining, the reduced lead set has a sensitivity of Anterior MI 56\%, compared to a retrained standard 12 lead set of $57 \%$, with specificity above $98 \%$ for both lead sets.
\end{abstract}

\section{Introduction}

Reduced lead sets use fewer leads than standard 12lead ECG; therefore can be more convenient in the situations of long-term and ambulatory monitoring. Most applications have converted the reduced lead sets to the standard 12-lead signals based on some regression algorithms, and then applied standard 12-lead algorithms to the converted data [1-3]. To do that, we would assume the morphology of relevant ECG segments in converted lead data do not have significant changes compared to the standard 12-lead system. For example, in ischemia detection cases, we would assume Q, ST and T wave segments do not have significant morphology changes after the conversion.

There are mainly two groups of reduced lead sets: group 1 uses a subset of standard 12-lead like 12RL (stand for 12 reduced lead) lead set used by GE, where standard leads I, II, v1 and v5 are used [1], or system uses standard leads I, II, v2, v5 [2]; Another groups used lead sets which are not from standard 12-lead, like EASI [3] or some other optimal lead sets under the context of different abnormalities [4].

The advantages of using a subset from standard 12lead set are (1) 8 of 12 leads are identical to the original 12 leads, so that physicians are familiar with majority of ECG morphology; (2) Large number of existing 12-lead ECG databases can be used for verification and validation, since complete reduced lead sets can be converted to 12 lead and then compared to actual sampled data. For the 4 derived leads, we would like to evaluate (a) how much differences exist between them and standard lead set? (b) If any significant differences exist, will they affect interpretation, either read by physicians or read by those computer programs which are trained with standard 12 lead data? (c) If some differences of morphology exist, could we retrain the criteria with converted reduced lead set to improve the interpretation performance of computerized interpretation algorithm.

Studies have shown some answers for question (a) and (b), that for majority ischemia cases physicians would be able to obtain correct interpretation based on the 12RL reduced lead set [1]. Therefore we would like to focus on the remaining question, i.e. how computerized algorithm can adapt to the new lead set?

In the study, we retrained interpretation algorithm to the new reduced lead data. For the purpose of retraining, there are quite a few learning algorithms can be used. Of those available learning algorithms, we selected a neural 
network algorithm and a binary decision tree algorithm. The input features to the models are ECG patterns from acute ischemia patients, especially those with acute anterior ischemia. The neural network (NN) models offer robust nonlinear regression for multiple inputs. Although NN models usually generate excellent classification results, they do not generate interpretive criteria. That's why we only use the NN model for a benchmark for the classification performance. At the meantime, the Binary decision tree (c4.5) is applied to the same data sets to generate not just classification results, also interpretive criteria. The combination of these two learning algorithms provided a fair assessment of the classification performance and clues of new ECG patterns that could be useful clinically.

\section{Methods}

\subsection{Input feature sets}

Standard 12-lead resting ECG data were used. Each ECG file includes 10 seconds simultaneously sampled 12-lead data. The median representative beat of the 10 seconds data was used for the further feature extraction. There are two groups of ECG features selected for acute inferior MI and anterior MI respectively. For anterior MI detection, the feature set include ST segment values of lead I, aVF, v1, v3, v5, T wave amplitudes of v3 and v5, and $\mathrm{Q}$ wave duration of $\mathrm{v} 3$. For inferior MI detection, the feature set include ST segment values of lead II, III, aVF, aVL, v2, v3, T wave amplitude of lead II, v2, v5, and Q wave amplitude of lead aVF.

\subsection{Convert the reduced lead set to 12-lead}

The reduced lead set $12 \mathrm{RL}$ is a subset of the standard 12-lead, consisting of all the limb leads and precordial lead v1 and v5 [1]. Another 4 precordial leads are derived based on the equations:

$$
\begin{aligned}
& \mathrm{V} 2=0.887330(\text { lead I) }-0.091160(\text { lead II })+ \\
& 1.578620(\text { lead V1) }+0.230214(\text { lead V5) } \\
& \mathrm{V} 3=0.245068(\text { lead I })+0.447773(\text { lead II })+ \\
& 1.147260(\text { lead V1 })+0.609744(\text { lead V5) } \\
& \mathrm{V} 4=0.111111(\text { lead I })+0.064849(\text { lead II })+ \\
& 0.465706(\text { lead V1) + 1.074230(lead V5) } \\
& \mathrm{V} 6=0.202721(\mathrm{lead} \mathrm{I})+0.038811(\mathrm{lead} \\
& 0.176913(\text { lead V1) }+0.594920(\text { lead V5) }
\end{aligned}
$$

The converted 12-lead set has the same frontal lead with the standard 12-lead, so that we could expect all the interpretations relying on frontal leads only to remain the same including axes measurements and inferior leads related ischemia.

\subsection{Training methods}

Two adaptive training methods, a neural network (NN) model with the structure of multi-layer perceptron and a binary decision tree based on C4.5 algorithm were used for their fast adapting speed and robustness in terms of handling large variations of the database.

\subsubsection{Neural networks}

The NN model adopted in the study has a multi-layer perceptron structure with 3 layers: Input, hidden, and output as shown in Figure 1 [5]. For the anterior MI detection, there are 8 input features as stated above. Therefore, 8 input units were used. For the inferior MI, 9 input units were used to match the 9 input features. For both models, the hidden layer of the $\mathrm{NN}$ model has 4 units, and the output layer has 3 units, each representing one out of 3 possible classes: MI, Unstable angina, or Non-ischemia.

Training method used is the Back-propagation algorithm [5]. After the $\mathrm{NN}$ model converged on the training set, final classification was taken from the maximum unit of the 3 output units.

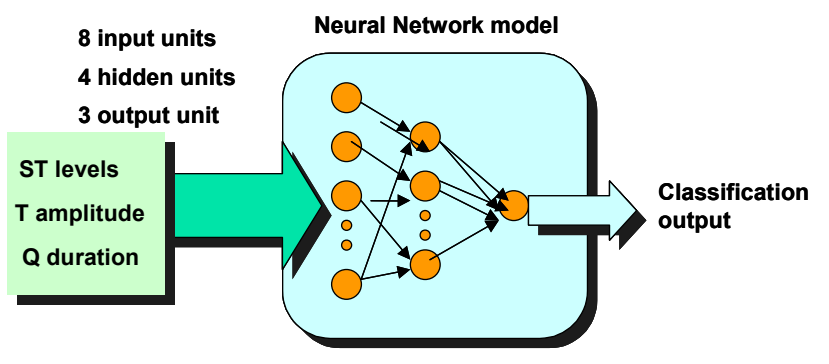

Figure 1. A three-layer neural network model for classification of ischemia.

\subsubsection{Binary decision tree $(\mathbf{C} 4.5)$}

The Binary decision tree model used in the study is based on the C4.5 by Quinlan [6]. In the model, decision tree is constructed based on the entropy gain provided by the selected branch. The tree model was originally designed for handing discrete data type. However, most ECG morphology features are continuous values. Here an ECG feature A is divided into many binary tests with outcome $\mathrm{A} \leq \mathrm{T}$ and $\mathrm{A}>\mathrm{T}$, based on comparing the feature value of A against a threshold value $T$.

The algorithm selects the feature with the highest information gain. If we assumes there are two classes, $\mathrm{P}$ (MI, p samples) and $\mathrm{N}$ (nonischemia, n samples ), then, 
the amount of information, needed to decide if an arbitrary example in $S$ belongs to $P$ or $N$ is defined as

$$
I(p, n)=-\frac{p}{p+n} \log _{2} \frac{p}{p+n}-\frac{n}{p+n} \log _{2} \frac{n}{p+n} \text { (5) }
$$

Assume that using feature A, a set $S$ will be partitioned into sets $\left\{S_{1}, S_{2}, S_{v}\right\}$, and expected information needed to classify objects in all subtrees $S_{i}$ is

$$
E(A)=\sum_{i=1}^{v} \frac{p_{i}+n_{i}}{p+n} I\left(p_{i}, n_{i}\right)
$$

The encoding information gain by branching on $A$ is

$$
\operatorname{Gain}(A)=I(p, n)-E(A)
$$

The number of trees of the model can increase vary fast with the increase of the number of input features. Therefore the decision trees need to be pruned in most cases. Another reason to prune the tree is to prevent overfiting which happened when inferring more structures than is justified by the training sets. In this study, pruning was done after original trees were formed retrospectively. Pruning may increase the classification error rate for training set, but has the advantages of better generalization on test set with simpler decision tree structure, meaning simpler final criteria for the classification of MI vs. unstable angina vs. non-ischemia.

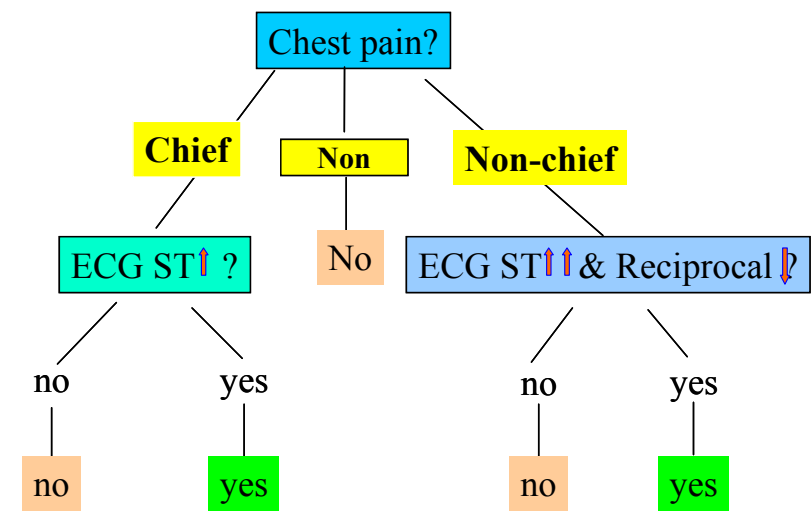

Figure 2. A simplified binary decision tree example for ischemia classification.

\subsection{Data sets}

The ECG data were collected from the two hospitals: Cardiac Care Unit (CCU) at Mayo Clinic, in Rochester, $\mathrm{MN}$, and the Milwaukee pre-hospital chest pain database at the Medical College of Wisconsin, in Milwaukee, WI.

Acute MI and acute ischemia ECG data were collected on residents of Olmsted County, MN with acute MI admitted to the Mayo Clinic CCU at Saint Mary's Hospital between January 1, 1988 and March 31, 1998 [7]. Acute MI was diagnosed (according to World Health Organization criteria) based on the appearance of abnormal new Q waves in serial electrocardiograms, or when two of the following were present: sequential ischemic ST-T changes on the ECG; increase in cardiac enzyme levels and typical chest pain lasting longer than 20 minutes.

The non-ischemic chest pain patients of Milwaukee Prehospital Chest Pain Database [8]. Prehospital 12-lead ECGs were sent by cellular telephone to a base station where they were interpreted in real time by a physician and subsequently digitally stored in a computer for later analysis. The final patient diagnosis was determined by hospital chart review by a team of trained nurse investigators and confirmed by a physician. Patients without evidence of acute MI or myocardial ischemia (angina pectoris) were classified as not having acute cardiac ischemia (non-ischemic).

In this study, total number of ECGs used includes 1187 acute inferior MI, 993 acute anterior MI, 617 unstable angina, and 887 non-ischemia. Sixty percent of them was used to form training set, and the other forty percent for testing set.

\section{Results}

\subsection{Tested on the criteria trained with the standard 12-lead data}

First, the interpretation algorithm trained with standard 12-lead data was used without modification.

For the Inferior MI, the sensitivity is $54 \%$ for the standard 12 -lead test set, and $53 \%$ for the reduced lead test set, while both have specificity of $99 \%$. Here the high specificity of the interpretation algorithm is expected from the field of practice. The performance of both lead sets for inferior MI interpretation are comparable.

For the anterior MI, the sensitivity is $46 \%$ for the standard 12-lead test set, and $37 \%$ for the reduced lead test set. Here the reduced sensitivity from the reduced lead set could be due to significant morphology changes for some derived anterior leads. Figure 4 shows an example of the difference in lead v3, where highlighted area indicates changes in ST-T segment.

\subsection{New criteria generated by training reduced set data}

For the retraining, the input features of anterior MI stated above were used. The classification of the output threshold was adjusted to reach the same high specificity $(>98 \%)$. After retraining, the reduced lead set has a 
sensitivity of Anterior MI $56 \%$, compared to a retrained standard 12-lead set of $57 \%$. The classification performances based on two lead sets are similar. The performances are also matched from both the neural network mode and the binary decision tree model.

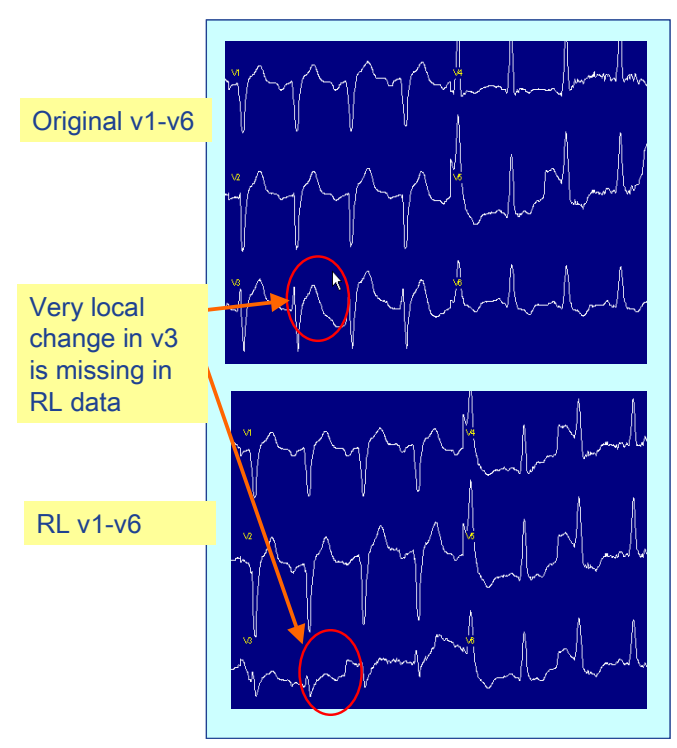

Figure 3. Difference of ST-T morphology between standard and reduced lead sets.

\subsection{Examples of rule inducted from the Binary decision tree}

Since the decision tree model also generates rules from the training set, we can evaluate some of them. For example, here are two rules induced from the anterior MI data set training:

(1) $\mathrm{ST}_{-} \mathrm{V} 3>131 \mathrm{uV}$ and $\mathrm{ST}_{-} \mathrm{I}<-50 \mathrm{uV}$ and Tamp_V5 $<-190$

(2) ST_V3 $>100 \mathrm{uV}$ and ST_AVF $<-19 \mathrm{uV}$, Tamp_V5 $<100 \mathrm{uV}$, Qdur_v3 $<=0$

The rule 1 shows that the ST elevation threshold is lower for the reduced lead than standard threshold of 200 $\mathrm{uV}$, while the condition on reciprocal ST depression of lead $\mathrm{I}$ and also the negative $\mathrm{T}$ wave compensate reduced ST level sensitivity. Rule 2 also shows the usefulness of the combination features.

\section{Discussion and conclusions}

Based on the results, we can see that the reduced lead set can achieve similar interpretation accuracy for inferior MI as those of standard 12-lead without any retraining. At the meantime, the reduced-lead specific algorithm can improve the anterior MI interpretation significantly compared to that using the standard 12-lead algorithm, and the reduced-lead set specific algorithm can adapt to the ECG morphology changes in derived anterior leads. The features of reciprocal ST level change and $\mathrm{T}$ wave become more important for the reduced lead sets for the purpose of ischemia detection.

For the purpose of the retraining interpretation algorithm from the new lead set, both neural network and binary decision tree models proved very effective. The former is very robust in terms of noise and variations, while the latter provides more transparent rules, which can be more acceptable in the clinical applications. The neural network model can also provide a benchmark for other adaptive learning methods to match even they are not directly used in the final released computerized interpretation algorithms.

\section{References}

[1] Drew BJ, Pelter MM, Brodnick DE, Yadav AV, Dempel D, Adams MG. Comparison of a new reduced lead set ECG with the standard ECG for diagnosing cardiac arrhythmias and myocardial ischemia. J Electrocardiol. 2002;35 Suppl:13-21.

[2] Nelwan SP, Kors JA, Meij SH, van Bemmel JH, Simoons ML. Reconstruction of the 12-lead electrocardiogram from reduced lead sets. J Electrocardiol. 2004 Jan;37(1):11-8.

[3] Rautaharju PM, Zhou SH, Hancock EW, Horacek BM, Feild DQ, Lindauer JM, Wagner GS, Pahlm O, Feldman CL. Comparability of 12-lead ECGs derived from EASI leads with standard 12-lead ECGS in the classification of acute myocardial ischemia and old myocardial infarction. J Electrocardiol. 2002;35 Suppl:35-9.

[4] Drew, C. Sommargren, D. Schindler, J. Zegre, K. Benedict, M. Krucoff, Novel electrocardiogram configurations and transmission procedures in the prehospital setting: effect on ischemia and arrhythmia determination?. J. of Electrocardiology, 2006; 39: S157S160 B.

[5] Masters T, Advanced algorithms for neural networks, John Wiley \& Sons, 1995

[6] Quinlan, JR, C4.5 : Programs for Machine Learning, Morgan Kaufmann, 1993

[7] Bybee KA, Wright RS, Williams BA, Murphy JG, Holmes DR Jr, Kopecky SL. Effect of concomitant or very early statin administration on in-hospital mortality and reinfarction in patients with acute myocardial infarction, Am J of Card.2001; 87:771-774.

[8] Aufderheide TP, ME Keelan, GE Hendley, et al. Milwaukee prehospital chest pain project - phase I: Feasibility and accuracy of prehospital thrombolytic candidate selection. Am. J. Cardiol. 1992;69:991.

Address for correspondence

Joel Xue

9900 Innovation Drive

Wauwatosa, WI 53226, USA 